\title{
Optimization of an autonomous robotic drilling system for the machining of aluminum aerospace alloys
}

\author{
Benjamin Pereira ${ }^{1}$ Christian Andrew Griffiths ${ }^{1} \cdot$ Benjamin Birch ${ }^{1} \cdot$ Andrew Rees $^{1}$
}

Received: 25 January 2021 / Accepted: 30 November 2021 / Published online: 15 December 2021

(c) The Author(s) 2021

\begin{abstract}
This paper aims to identify the capability of a highly flexible industrial robot modified with a high-speed machine spindle for drilling of aluminum 6061-T6. With a focus on drilling feed rate, spindle speed, and pecking cycle, the hole surface roughness and exit burr heights were investigated using the Taguchi design methodology. A state of the art condition monitoring system was used to identify the vibrations experienced during drilling operation and to establish which robot pose had increased stiffness, and thus the optimum workspace for drilling. When benchmarked against a CNC machine the results show that the $\mathrm{CNC}$ was capable of producing the best surface finish and the lowest burr heights. However, the robot system matched and outperformed the $\mathrm{CNC}$ in several experiments and there is much scope for further optimization of the process. By identifying the optimum pose for drilling together with the idealized settings, the proposed drilling system is shown to be far more flexible than a CNC milling machine and when considering the optimized drilling of aerospace aluminum this robotic solution has the potential to drastically improve productivity.
\end{abstract}

Keywords Automation $\cdot$ Robotics $\cdot$ Drilling $\cdot$ Machine spindle $\cdot$ Aerospace alloys

\section{Introduction}

The aerospace manufacturing industry is always looking for ways to reduce cost, improve quality, and reduce lead time. Drilling within the aerospace sector has always been very important for the manufacture of airplanes and is the most commonly performed task in machining aerospace components. The Boeing 747 contains roughly 3 million fasteners with roughly 40,000 rivets on each wing of a Boeing [1] with Boeing drilling roughly 1.1 million holes per day [2]. In a bid to improve efficiency, the implementation of automation within the automotive sector has increased from 20 to $80 \%$ [3]. Despite the similarities between aerospace and automotive the drive for automation has not been witnessed within the aerospace sector despite the benefits that could be gained [4].

Airbus and Boeing released their global market forecast for 2018-2037 in July 2018. The reports show a predicted growth of $3.5 \%$ of new airplane deliveries per annum [5]. In

Christian Andrew Griffiths

c.a.griffiths@swansea.ac.uk

1 College of Engineering, Swansea University, Swansea SA2 8PP, UK addition, during the same time period there is expected to be a $4.4 \%$ annual growth in air traffic [6]. Additional sources of forecasts from Airbus and Boeing highlight a continuing demand for civil aircraft between 2015 and 2034 whereby aircraft volumes will increase from 19,000 to 38,500 [7]. This data demonstrates the expected growth that will be witnessed within the aerospace sector moving forward. To meet the growing demand for aircraft manufacture automation has the potential to increase both productivity and component quality within the aerospace sector.

\subsection{Drilling operations}

Within the aerospace sector assembly work is commonly highly physical and can be monotonous. Typically, the assembly of an aircraft fuselage or wing consists of skin sheets being mounted onto supporting structures that are subsequently connected through riveting [8]. The manual riveting operation requires a pre-drilling drilling operation which can cause several potential health risks related to fatigue, dust, noise, and vibration [9]. In addition, the sector traditionally has inherent high labor turnovers, training costs, and recruitment difficulties due to the nature of the machining and assembly procedures used [10]. Duquette 
et al. identified that at least $30 \%$ of workers found that the tools used to assemble aircrafts introduced back pain after prolonged use [10]. Also, a study by Menegon et al. on 522 aircraft assembly workers reported that pain, discomfort, and numbness were experienced in body regions such as neck, shoulders, and knees which correlated to employees seek leave of absence from work [11]. In addition, within the sector the quality of drilling operations suffers from inconsistency. Typically, quality is higher at the beginning of a shift when compared to the work produced at the end of a shift. This variation is quality can be attributed to operator fatigue and human constraints which highlights the urgent requirement to introduce more automation into labor-intensive activities. One challenge for automation is the quality of the drilled holes. The precision of the connecting holes determines the riveting quality. Any introduction of imprecision will result in bending stress being introduced at the joints [12]. This can be detrimental to the fatigue life of the aircraft with $80 \%$ of structural failures being attributed due to fatigue damages from the connecting holes of the aircraft structures [13, 14]. Also, tight hole tolerances are required; therefore, any manual drilling with inherent poor hole quality consistency will require subsequent rework [15]. These errors are a concession to the original specification, therefore increases the manufacturing costs to rectify [16].

Vibrations during the drilling process often lead to chatter which in turn causes poor hole quality, increased noise levels, and potential damage to the component [17]. Tao et al. identified the importance of identifying chatter as early as possible to improve hole quality [18]. When considering different feed rates $\left(F_{r}\right)(0.9-7.8 \mathrm{~mm} / \mathrm{s})$ and the spindle speeds $\left(S_{s}\right)$ (1800-3600 RPM) the research showed that increasing the $S_{s}$ resulted in decreased stability due to rapid increase of the amplitude of vibrations, leading to higher levels of chatter. Recent evidence suggests that provided the correct machining parameters are identified and used, increased chatter levels can be avoided [19-21]. These studies go on to state that at lower $S_{s}$ rates the chatter levels can be reduced due to the stabilizing effect of the process damping. The drilling parameters also affect the surface roughness $\left(R_{a}\right)$ [22]. Zhang showed that the application of different types of drills and the setup of the different drilling parameters can have a major effect on the resulting $R_{a}$ of the hole [23]. Chen identified that $F_{r}$ is an important machining parameter which influences both the accuracy and surface quality of drilled holes and that an adaptive material removal rate increases machining accuracy [24]. Huang et al. provided an optimization analysis of the drilling parameters while dry machining aluminum 6061 alloy [25]. Using a Taguchi's signal/noise ratio and grey relational analysis method the investigation concluded that the optimal drilling parameters for the desired $R_{a}$ are $S_{s}$ of $3000 \mathrm{RPM}$ and $F_{r}$ of $0.2 \mathrm{~mm} / \mathrm{rev}$.
While the optimal drilling parameters for the desired burr height are $S_{s}$ of $2500 \mathrm{RPM}$ and $F_{r}$ of $0.15 \mathrm{~mm} / \mathrm{rev}$ [25].

\subsection{Hole burrs}

Hole burrs are an undesired by-product produced during the drilling process. They consist of plastically deformed material at the entry and exit peripheries. Typically, a burr is an accumulation of plastically deformed work material, and if left unremoved, burrs can impact the overall quality and life of a product [26, 27]. In 2004, Kishawy et al. demonstrated that hole burrs can have significant effects on the fatigue life of aluminum aerospace alloys. The study highlighted that test pieces with burrs were found to have significantly reduced fatigue life by up to two orders compared to nonburred specimens [28]. Deburring is classed as a non-valueadded manufacturing process that can require a significant amount of production time especially on large structures [29]. Studies on the influencing factors of burr formation show that the burr height can be significantly influenced by the workpiece material, tool geometry, and wear and machining parameters [30, 31]. L. Jie observes that burrs cannot be completely prevented by changes in feed, speed, or tool geometry in isolation. The research concluded that burr height can be minimized significantly by selecting the appropriate drilling parameters [31]. In a study by Lee the influence of machine parameters on the resulting thrust force $\left(F_{t}\right)$ in drilling was investigated [32]. The research demonstrates that the resulting thrust force determines the amount of material that undergoes plastic deformation during the final stage of drilling [32]. Further studies on burr formation have concluded that burr height increases as a function of drill wear. Also burr height can be reduced through dry machining [33].

\subsection{Automation of drilling}

Drilling in aerospace is predominantly done using Advanced Drilling Units (ADUs) with some manufacturers moving to Light Electric Drilling Units (LEDUs). In an investigation into the application of industrial robotics for the assembly of commercial aircraft components, Durham found that hole quality can be controlled by adjusting the $F_{r}, S_{s}$, and pecking cycle $\left(P_{c}\right)$ [34]. The research showed the time savings obtainable through the integration of automation within the assembly and manufacture of aerospace components and identifies the potential economic savings of automation. Furthermore, Durham demonstrated that even a 1-s reduction in takt time per hole on the 8000 exterior holes required in a fuselage would save $13 \mathrm{~h}$ of operation [34]. Historically, the reluctance to use automation within the aerospace sector has been attributed to the poor accuracy of early generation robots. Poor arm and joint stiffness lead to higher levels of 
vibrations. Traditional CNC machines have a stiffness of greater than $50 \mathrm{~N} / \mu \mathrm{m}$, whereas a typical articulated robot usually has less than $1 \mathrm{~N} / \mu \mathrm{m}$. Zhang et al. state that the difference in stiffness between the two machines is a major hurdle preventing the adoption of robots within industry [17]. In recent years, there have been vast improvements in manufacturing robots and specifically the 6-axis manipulator-type robot resulting in higher accuracy and repeatability $[35,36]$. They have become increasingly stiffer which correlate to higher accuracy machining when compared to older generations of robots. This has permitted machine spindles to be used as end effectors and thus allowing for the possibility of highly flexible automated drilling operations. However, manufacturing robots have some drawbacks; the "Fuselage Automated Upright Build" process (FUAB) was abandoned by Boeing in 2019 as the robots proved painful to set up and were error-prone resulting in fuselages having to be finished by hand [37]. Despite the challenges research has demonstrated the benefits of robotics utilization. In particular, Yin et al. concluded in their study that improving the KUKA KR500-2's stiffness lead to better accuracy of countersink drilling depth [38]. Also, by reducing vibrations during the automated drilling process tool wear and tool breakage is decreased. In addition, dimensional quality is improved and surface roughness is reduced $[39,40]$.

This paper will investigate the utilization of a state-ofthe-art Kuka KR-16 manipulator with a high-speed spindle and drill end effector for the machining of aerospace AA601 aluminum alloy. The study will utilize an aerospace compliance standard for hole accuracy and burr height drilling operations for dry machining applications [41]. The main aims are to optimize dry machining parameters through the use of the Taguchi design of experiment (DOE). The resulting machining responses of surface roughness, burr height, and concentricity of the holes produced will be empirically compared to the results obtained from a conventional 3-axis $\mathrm{CNC}$ milling machine. The research will then investigate the Kuka machining setup, in particular the resulting vibration as a function of machining position. This will be followed by a DOE to establish the machining parameters that have the largest effect on the quality of holes. Section 3 will analyze the results recorded for these experiments and benchmark the robotic machining against the $\mathrm{CNC}$ system. Finally, conclusions will be made on the potential of this method of flexible automation for machining aerospace components.

\section{Experimental}

\subsection{Drilling parameters}

The holes to be analyzed for this research are 8-mm diameter and 8-mm deep; drilling parameters for these dimensions
Table 1 L9 orthogonal array

\begin{tabular}{llll}
\hline Experiment & $F_{r}$ & $S_{s}$ & $P_{c}$ \\
\hline 1 & 1 & 1 & 1 \\
2 & 1 & 2 & 2 \\
3 & 1 & 3 & 3 \\
4 & 2 & 1 & 2 \\
5 & 2 & 2 & 3 \\
6 & 2 & 3 & 1 \\
7 & 3 & 1 & 3 \\
8 & 3 & 2 & 1 \\
9 & 3 & 3 & 2 \\
\hline
\end{tabular}

have been identified. The $F_{r}$ range used is between 200 and $600 \mathrm{~mm} / \mathrm{min}, S_{s}$ of 1800-4000 RPM, and the number of passes of a pecking cycle $\left(P_{c}\right)$ of $2-6 \mathrm{~mm}$ at a time. $P_{c}$ is defined as the machining method that is used in the drilling processes to prevent swarf from building up while drilling holes. The pecking depth improves tool life but each additional cycle increases the drilling takt time. In this research a robotic-based $P_{c}$ that considers different pecking depths will be used to gain an insight into whether or not the surface roughness of the holes is influenced. The robot and CNC canned pecking cycle will also be compared.

\subsection{Design of experiments}

Within the study an L9 Taguchi array design with 3 factors and 3 levels will be used and a total of 3 experimental repeats will be undertaken (Table 1). For the drilled material, aluminum 6061-T6 has been selected. The alloy is used widely within the aerospace sector in the skin of aircrafts due to its excellent mechanical properties of hardness, weldability, corrosion resistance, and relatively low cost. The experimental parameters used to conduct the drilling experiments are detailed in Table 2.

\subsection{Kuka KR-16 Robotic drilling system}

The study was conducted using a KUKA KR16 six-axis industrial robot (Fig. 1). The robot has a positional repeatability accuracy of $\pm 0.04 \mathrm{~mm}$, payload of $16 \mathrm{~kg}$, and a maximum reach of $1610 \mathrm{~mm}$. The end effector used within the study is a Zimmer HFL04-103-01-A-00 high-speed machine spindle. The machining spindle is $3.6 \mathrm{~kW}$ with a maximum

Table 2 Process parameters

\begin{tabular}{llll}
\hline Experimental parameters & Level 1 & Level 2 & Level 3 \\
\hline$F_{r}(\mathrm{~mm} / \mathrm{min})$ & 200 & 300 & 400 \\
$S_{s}(\mathrm{RPM})$ & 2000 & 2500 & 3000 \\
$P_{c}$ & 2 & 3 & 4 \\
\hline
\end{tabular}




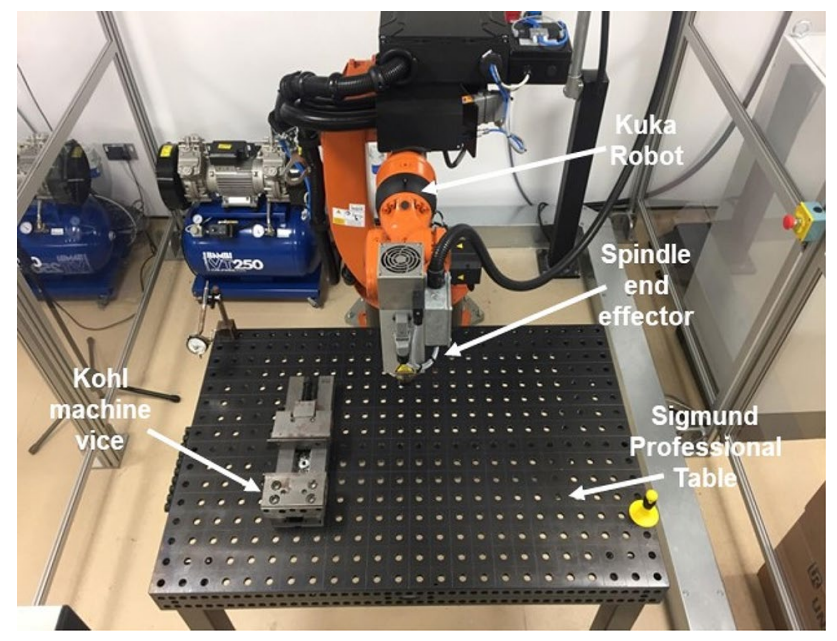

Fig. 1 Kuka KR16 and Zimmer machine spindle and table

spindle speed of 24,000 RPM. A collet is used to accurately hold the $8-\mathrm{mm}$ high-speed steel drill. The workpiece is held in a Kohl machine vice bolted to a Sigmund Professional table. The table bed provides a $1200 \times 800 \times 100-\mathrm{mm}$ plasma-nitrided steel work space and is bolted to the floor to ensure that it is rigid. To benchmark the Kuka KR-16 Robotic drilling system a XYZ SMX/RMX 3500 CNC machine with similar machine parameters was used (Fig. 2).

\subsection{Vibration testing within robot workspace}

Drill chatter and vibration can have a large effect on the quality of the hole produced. Therefore, in order to try and ensure that the holes being drilled experience the least amount of chatter, vibration tests were undertaken to

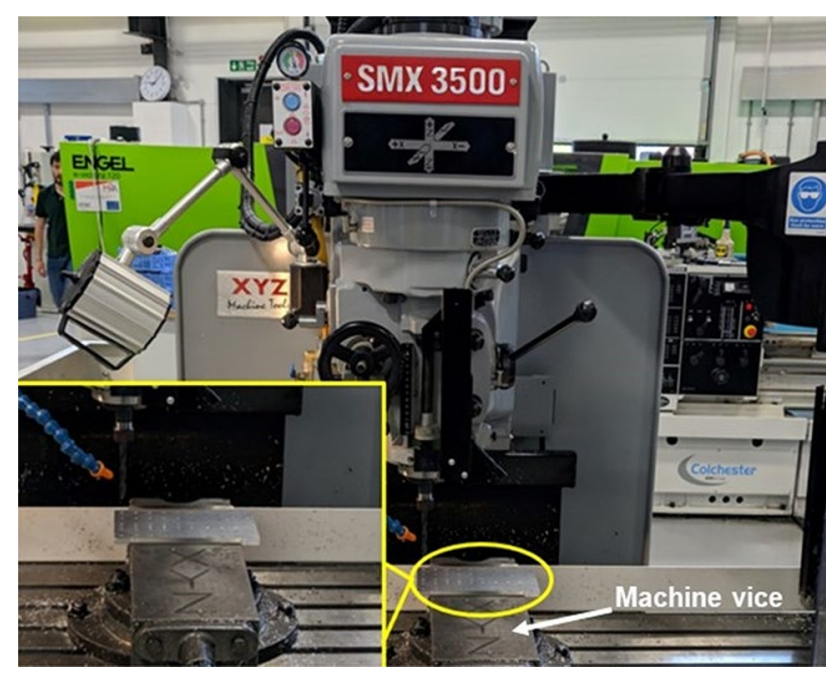

Fig. 2 XYZ SMX/RMX $3500 \mathrm{CNC}$ measure which sections of the robot workspace had the lowest vibration amplitude. To establish the vibrations of the robot system a condition monitoring system is used. Two SignalCalc Ace Vibration sensors are connected to DAQ (V-A) and SignalCalc software is used to record data from the accelerometers. The first accelerometer was placed on the side of the machine head to measure the vibrations in the $x$ plane (Fig. 3). The second accelerometer was placed on the top of the machine head to measure the $z$ plane vibrations. The robot was then driven to 99 pre-defined positions within the workspace table and a condition monitoring system measured the acceleration and displacement while at each location. The vibration tests of the workspace were run firstly with the spindle off. However, to ensure that the pose stiffness results are accurate the test are reperformed but with the spindle active. The robot moves to the same 99 static positions but this time with the following spindle RPMs: 2000, 2500, 3000, 5000, 10,000, and 20,000 RPM. The range of RPMs is used to gauge how the $S_{s}$ effect the vibrations of the robot machine spindle. The results from the initial vibration tests were analyzed in order to see which location within the workspace had the lowest amplitude of vibrations and thus the stiffest pose of the Kuka KR16 while machining. Once this is established the machine vice is then set to this position to conduct the experiments.

\subsection{Metrology}

Pretrials identified that the burrs from the experiments are to be classified as breakouts (negative burrs). Due to the plastic deformation of a burr, non-destructive measurements are preferred as the specimen is not further deformed during the measurement. Once the holes were drilled, the procedure used was photographed to allow them to be examined and ranked according to the severity of the burr. The test piece is placed under the Keyence VHX-1000 digital microscope to observe the hole exit burrs. In order to generate focused images, depth composition was used image the different burr heights. After the burr measurements had been taken, the part was cut down the middle and the specimen was then put under the Veeco Wyko NT9300 white light interferometer to measure surface roughness.

\section{Experimental results}

\subsection{Vibration results}

\subsubsection{Vibration results with the spindle off}

Most lead through positional programming by the engineer is based on identifying a specific work space and then finding the optimum robot poses within this space. For this 
Fig. 3 Condition monitoring setup

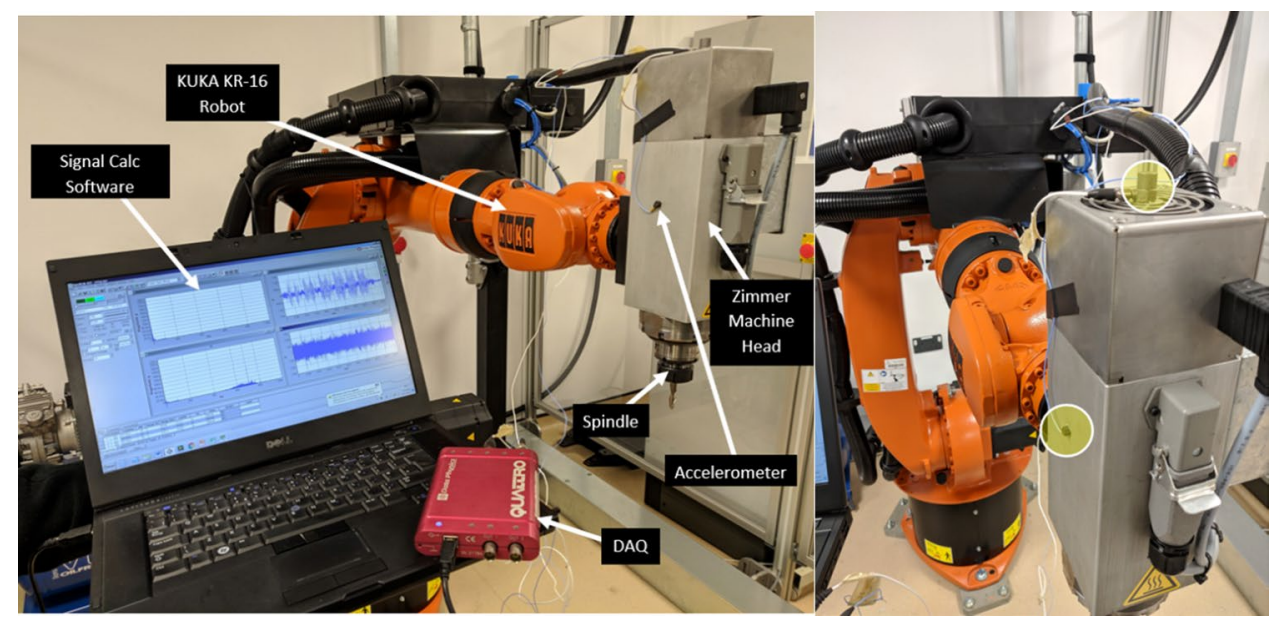

research 99 drill positions on the $1200 \times 800 \times 100-\mathrm{mm}$ Sigmund table were identified and vibration experiments were performed without the spindle switched on. The probability plot of the results in Fig. 4 shows a normal distribution with a mean of $0.0037 \mathrm{~mm}$. In order to identify regions with increased vibration Fig. 5 shows a map of the robot vibrations experienced within the drilling workspace. The probability plot is used to identify results above $0.005 \mathrm{~mm}$, and these results are highlighted in grey in Fig. 5. The allwhite blocks are the locations within the workspace that the robot could not reach due to the pose limitations. The vibration data indicates that although the robot is static in terms of drilling there are still vibrations and dynamic responses passing through the robot. The kinematics of the robot are based on Denavit Hartenberg parameters, and there are rules that the robot must follow in order to rotate coordinate frames. Thus, the positions of each of the 6 axes are based on a default kinematics unless modified by the operator. The variation in vibration within the work space shows that the kinematic chain of 6-axis articulated robots are stiffer in certain poses but there are no clear pose regions that are better over the test space.

\subsubsection{Vibration results with the spindle on}

The Zimmer HFL04-103-01-A-00 machine spindle is capable of operating up to 24,000 RPM so it was decided that the maximum, minimum, and average vibrations up to this RPM range would be measured. Figure 6 shows that the vibration of the entire workspace at various RPMs does not fluctuate dramatically between 2000 and 10,000 RPM. However, there is an increase of $40 \%$ between 3000
Fig. 4 Probability plot for vibration amplitude $(\mathrm{mm})$ with the spindle off

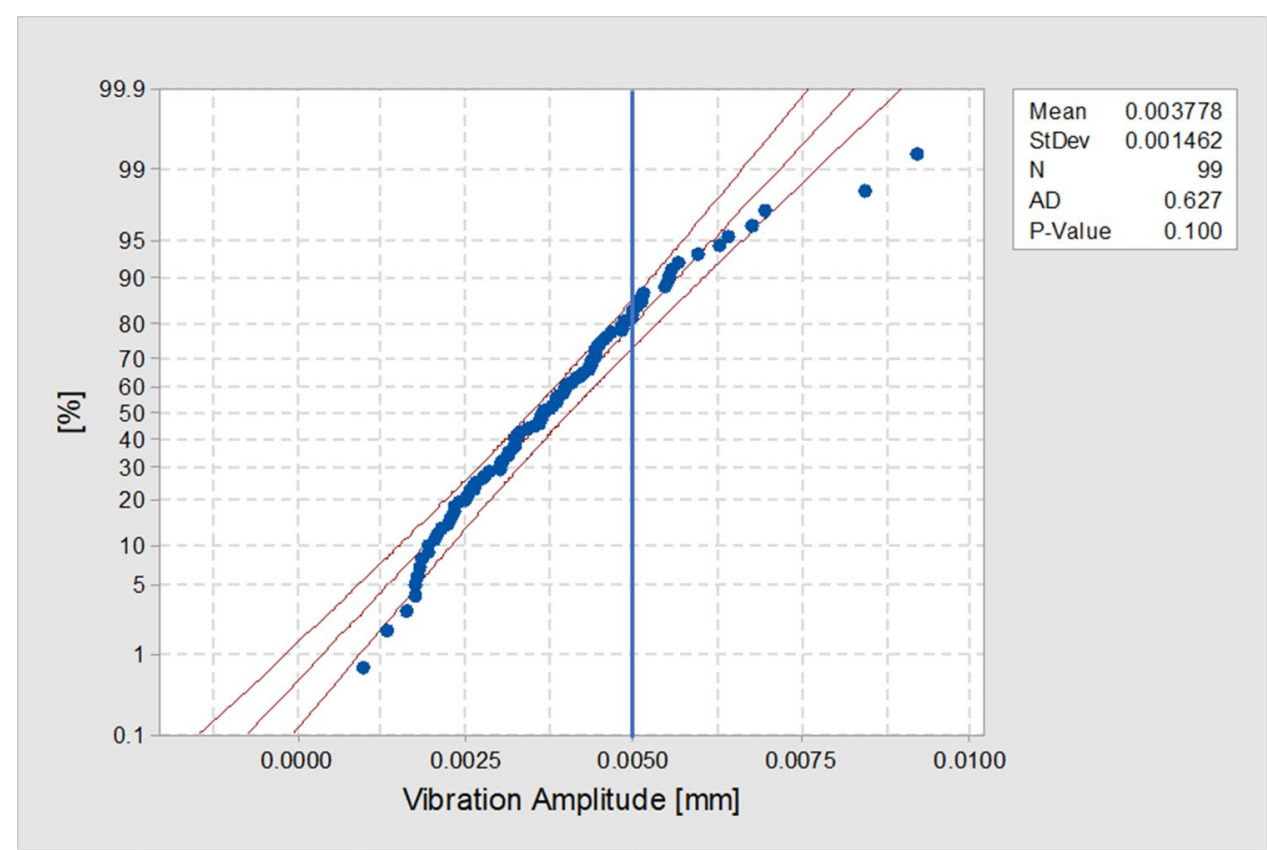




\begin{tabular}{|l|l|l|l|l|l|l|l|l|l|l|l|l|l|}
\hline 1 & & & & 0.004 & 0.004 & 0.003 & 0.002 & 0.003 & 0.005 & 0.003 & & & \\
\hline 2 & & & & 0.001 & 0.004 & 0.003 & 0.004 & 0.002 & 0.005 & 0.005 & & & \\
\hline 3 & & & 0.003 & 0.005 & 0.004 & 0.004 & 0.004 & 0.005 & 0.005 & 0.003 & 0.002 & & \\
\hline 4 & & 0.005 & 0.004 & 0.004 & 0.003 & 0.004 & 0.003 & 0.003 & 0.004 & 0.004 & 0.004 & 0.002 & \\
\hline $\mathbf{5}$ & 0.007 & 0.006 & 0.004 & 0.005 & 0.004 & 0.006 & 0.006 & 0.004 & 0.006 & 0.003 & 0.005 & 0.005 & 0.004 \\
\hline $\mathbf{6}$ & 0.002 & 0.002 & 0.003 & 0.003 & 0.008 & 0.004 & 0.002 & 0.006 & 0.007 & 0.005 & 0.004 & 0.009 & 0.006 \\
\hline 7 & 0.004 & 0.002 & 0.003 & 0.003 & 0.003 & 0.002 & 0.004 & 0.004 & 0.002 & 0.003 & 0.002 & 0.004 & 0.003 \\
\hline $\mathbf{8}$ & 0.003 & 0.004 & 0.003 & 0.001 & 0.004 & 0.005 & 0.005 & 0.005 & 0.002 & 0.003 & 0.002 & 0.002 & 0.002 \\
\hline $\mathbf{9}$ & 0.004 & 0.004 & 0.003 & 0.002 & 0.002 & 0.003 & 0.003 & 0.004 & 0.004 & 0.004 & 0.004 & 0.005 & 0.002 \\
\hline & $\mathbf{1}$ & $\mathbf{2}$ & $\mathbf{3}$ & $\mathbf{4}$ & $\mathbf{5}$ & $\mathbf{6}$ & $\mathbf{7}$ & $\mathbf{8}$ & $\mathbf{9}$ & $\mathbf{1 0}$ & $\mathbf{1 1}$ & $\mathbf{1 2}$ & $\mathbf{1 3}$ \\
\hline
\end{tabular}

Fig. 5 Vibration amplitude $(\mathrm{mm})$ heatmap of the workspace with the spindle off

and 5000 RPM, and an increase of $655 \%$ from 10,000 to 20,000 RPM. The findings comply with the work of Tao et al. [10]. In their paper they observed that when the spindle speed was increased, the stability decreased. The potential for speeds $>3000$ RPM to have a high vibration amplitude means that the stability of the robot will negatively influence the quality of the holes. This finding was

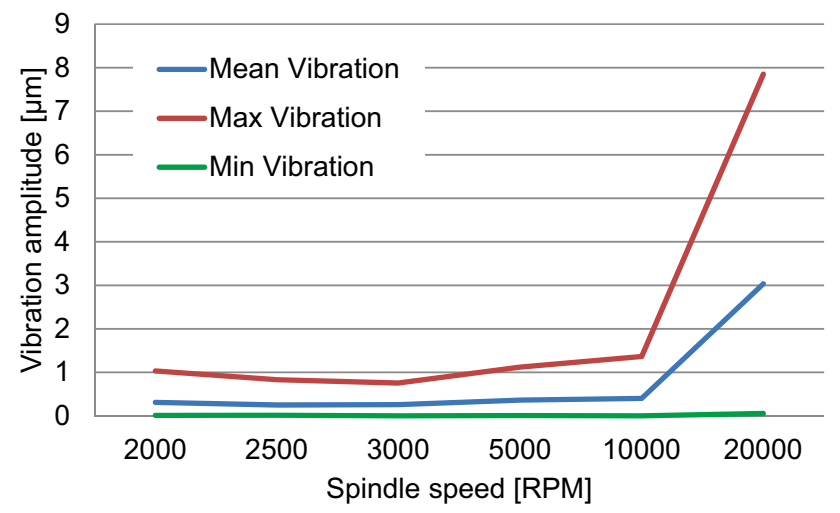

Fig. 6 Vibration amplitude for different spindle speeds important in terms of the capability of the robot and it was decided that only speeds below this will be investigated further in this study.

\subsubsection{Vibration results with the spindle at the selected operation range}

Following on from the spindle off experiments the same was conducted with the spindle on. The purpose for this is to identify if the established low-vibration poses (Fig. 4) are influenced by the centrifugal force from the machine spindle speeds. The probability plot of the results in Fig. 7 shows that the mean vibration amplitude is $0.274 \mathrm{mmm}$ and also shows that the distribution is not normal. Notably there are five results above $0.50 \mathrm{~mm}$ that influence the distribution. Figure 8 shows a map of the average vibrations in each position within the table workspace across the following RPMs: 2000, 2500, and 3000. The maximum vibration value $\left(V_{m}\right)$ was taken at each location to identify the optimum areas for machining. The map was obtained by taking the $V_{m}$ value at each position at each rpm then taking a vibration average $\left(V_{a}\right)$ across the three RPMs at each position and plotting the average value on one heat map. The lowest $V_{a}$ average 
Fig. 7 Probability plot for vibration amplitude $(\mu \mathrm{m})$ with the spindle on

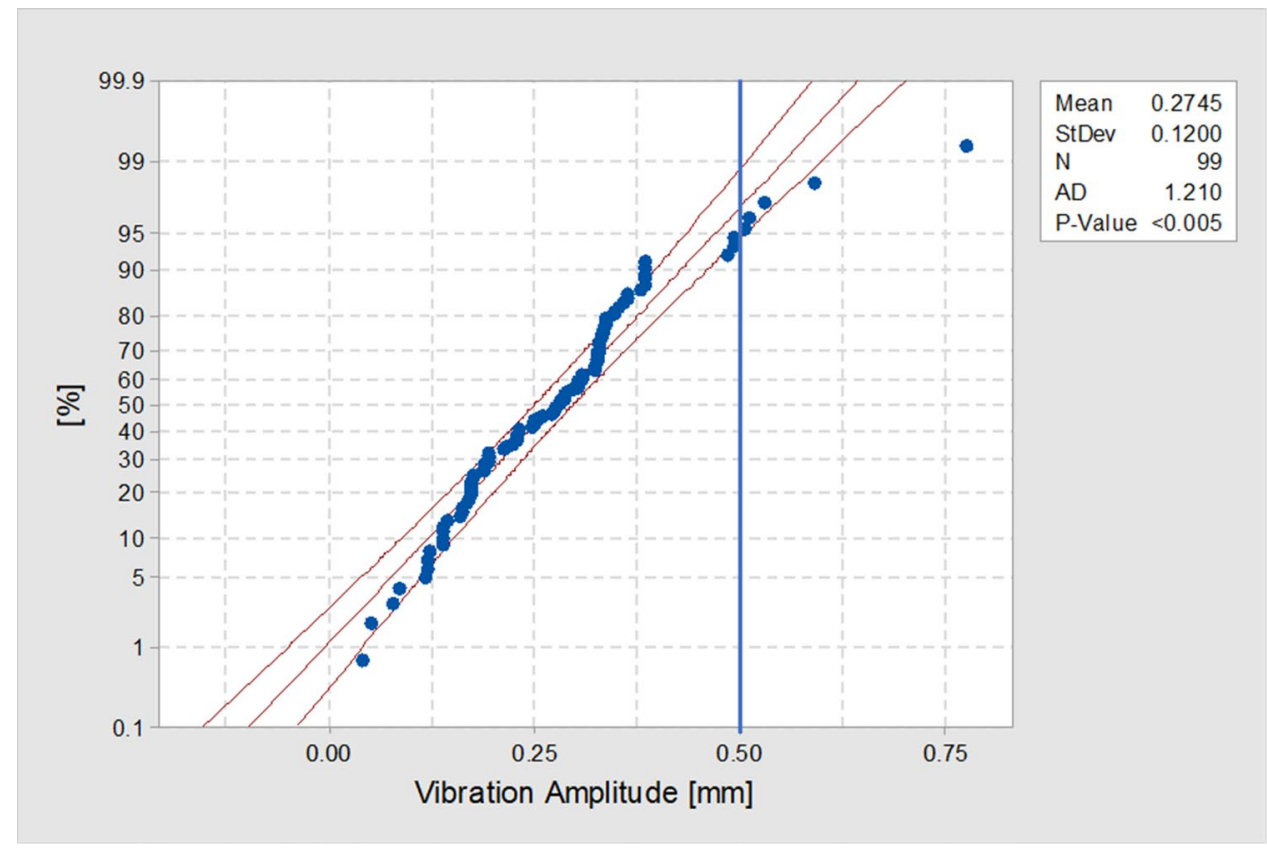

vibration experienced $(0.038 \mathrm{~mm})$ is in block in $(4,7)$. All results above $0.50 \mathrm{~mm}$ are shown in grey and the area of highest $V_{a}(0.77 \mathrm{~mm})$ is shown in block $(8,8)$. The all-white blocks are the locations within the workspace that the robot

\begin{tabular}{|l|l|l|l|l|l|l|l|l|l|l|l|l|l|}
\hline $\mathbf{1}$ & & & & 0.194 & 0.334 & 0.119 & 0.293 & 0.302 & 0.357 & 0.352 & & & \\
\hline $\mathbf{2}$ & & & & 0.336 & 0.247 & 0.193 & 0.384 & 0.334 & 0.119 & 0.194 & & & \\
\hline $\mathbf{3}$ & & & 0.385 & 0.385 & 0.173 & 0.117 & 0.379 & 0.273 & 0.248 & 0.280 & 0.280 & & \\
\hline $\mathbf{4}$ & & 0.187 & 0.187 & 0.136 & 0.136 & 0.306 & 0.306 & 0.167 & 0.346 & 0.277 & 0.328 & 0.211 & \\
\hline $\mathbf{5}$ & 0.326 & 0.325 & 0.362 & 0.383 & 0.326 & 0.326 & 0.168 & 0.285 & 0.302 & 0.192 & 0.187 & 0.223 & 0.121 \\
\hline $\mathbf{6}$ & 0.336 & 0.510 & 0.492 & 0.529 & 0.492 & 0.226 & 0.175 & 0.175 & 0.323 & 0.323 & 0.276 & 0.084 & 0.361 \\
\hline $\mathbf{7}$ & 0.327 & 0.173 & 0.323 & 0.038 & 0.227 & 0.138 & 0.077 & 0.049 & 0.227 & 0.229 & 0.229 & 0.161 & 0.161 \\
\hline $\mathbf{8}$ & 0.172 & 0.287 & 0.331 & 0.331 & 0.172 & 0.137 & $\mathbf{0 . 5 9 1}$ & $\mathbf{0 . 7 7 5}$ & 0.171 & 0.383 & 0.347 & 0.301 & 0.253 \\
\hline $\mathbf{9}$ & 0.308 & 0.160 & 0.142 & 0.336 & 0.286 & 0.484 & 0.505 & 0.258 & 0.250 & 0.214 & 0.327 & 0.327 & 0.269 \\
\hline & $\mathbf{1}$ & $\mathbf{2}$ & $\mathbf{3}$ & $\mathbf{4}$ & $\mathbf{5}$ & $\mathbf{6}$ & $\mathbf{7}$ & $\mathbf{8}$ & $\mathbf{9}$ & $\mathbf{1 0}$ & $\mathbf{1 1}$ & $\mathbf{1 2}$ & $\mathbf{1 3}$ \\
\hline
\end{tabular}

Fig. 8 Mean vibration amplitude $(\mu \mathrm{M})$ heatmap of the workspace with the spindle on (2000-3000 RPM) 
could not reach due to the pose limitations. The vibration data indicates that the $V_{a}$ observed across the three RPMs is 40 times larger than when the spindle was off, and the $V_{m}$ observed across the three RPMs is 84 times larger than when the spindle was off. This shows that the robot experiences significant vibrations when the spindle is turned on. The vibration map does provide the programmer with a wide range of regions with below $V_{m}$ allowing for an optimized drilling workspace.

\subsection{Burr results}

\subsubsection{Main distribution of the exit burr heights from the CNC and Kuka systems}

To compare both drilling systems nine burr height measurements were made for each of the experiments (Fig. 9). The measured burr heights for all the CNC experiments show that the mean height is $0.9 \mathrm{~mm}$. The minimum observed average height $(0.15 \mathrm{~mm})$ is observed in experiment 8 and as this experiment also has the lowest deviation ( $\sigma$ of 0.021 ), this making it the optimum CNC setting. The settings for experiment 2 produced the highest bur heights and largest deviations with $1.85 \mathrm{~mm}$ and $\sigma$ of $1.55 \mathrm{~mm}$, respectively. There is a reasonable consistency in burr height and deviation for experiments $3-7$.

The mean measured burr height for the Kuka holes for all experiments is $1.07 \mathrm{~mm}$; this is $16 \%$ less than the $\mathrm{CNC}$ average. The best setting is experiment 1 as it produces an average minimum observed burr height of $0.29 \mathrm{~mm}$ with a low $\sigma$ of $0.021 \mathrm{~mm}$. The maximum is $3.11 \mathrm{~mm}$ from experiment 3 , which is the highest exit height of all experiments and has a wide deviation of measured heights. Except for experiment 3 there is a reasonable distribution except for the high height observed with experiment 3 and consistency in the results between 4 and 9.

An important observation to be made when considering both systems is that although the average burr height for the CNC system is better throughout the 9 experiments, the Kuka system can produce lower exit burrs as demonstrated by experiments $1,2,5,6,7$, and 9 . It can also be seen that for nearly all experiments the Kuka drilling results have a lower variation in measured burr height. This suggests that the settings used on the Kuka system produce exit burrs that are more consistent and that the burrs may not be significantly influenced by the overall stiffness of the systems.

\subsubsection{Main effects of the exit burr heights from the CNC and Kuka systems}

Table 3 shows the main effects of the parameters on burr heights for both drill systems. Based on the results the $P_{c}$ has the largest influence on both. The ranking is showed by the difference between the lowest and highest $(\delta)$ of $0.49 \mathrm{~mm}$ and $1.04 \mathrm{~mm}$, respectively. For both systems an increase in the $P_{c}$ increases the burr height (Figs. 10 and 11). $F_{r}$ is ranked second and for the CNC the burr height is reduced as the $F_{r}$ is increased. For the Kuka the $F_{r}$ increase from 200 to $300 \mathrm{~mm} / \mathrm{min}$ reduces the burr height by $0.96 \mathrm{~mm}$ but there is a marginal height increase from 300 to $400 \mathrm{~mm} / \mathrm{min}$.
Fig. 9 Burr heights for $\mathrm{CNC}$ and Kuka drilled holes

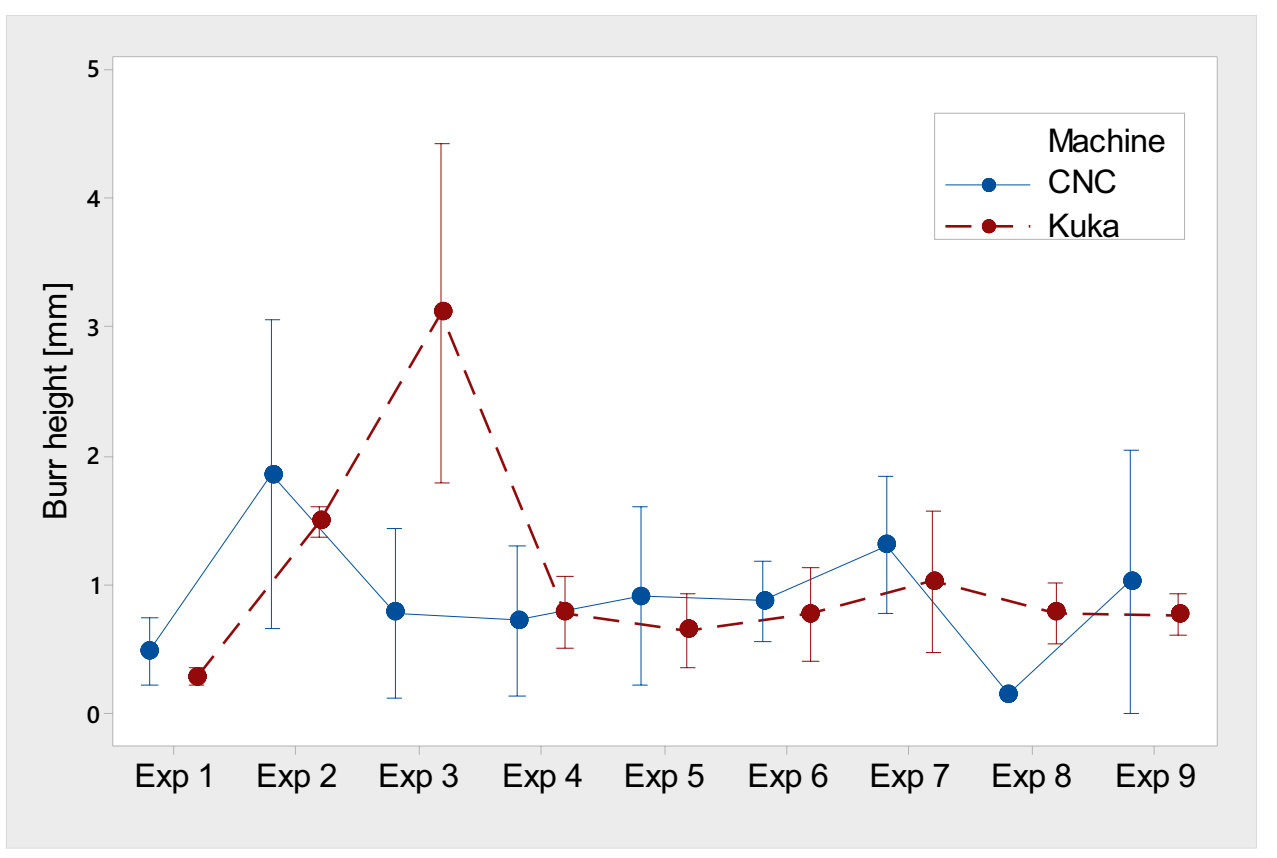


Table 3 Exit burr height main effect results

\begin{tabular}{|c|c|c|c|c|c|c|}
\hline \multirow[t]{2}{*}{ Level } & \multicolumn{3}{|l|}{$\mathrm{CNC}$} & \multicolumn{3}{|l|}{ Kuka } \\
\hline & $F_{r}$ & $S_{s}$ & $P_{c}$ & $F_{r}$ & $S_{s}$ & $P_{c}$ \\
\hline 1 & $1.04 \mathrm{~mm}$ & $0.84 \mathrm{~mm}$ & $0.50 \mathrm{~mm}$ & $1.63 \mathrm{~mm}$ & $0.78 \mathrm{~mm}$ & $0.56 \mathrm{~mm}$ \\
\hline 2 & $0.83 \mathrm{~mm}$ & $0.97 \mathrm{~mm}$ & $0.94 \mathrm{~mm}$ & $0.67 \mathrm{~mm}$ & $0.87 \mathrm{~mm}$ & $1.01 \mathrm{~mm}$ \\
\hline 3 & $0.57 \mathrm{~mm}$ & $0.64 \mathrm{~mm}$ & $1.00 \mathrm{~mm}$ & $0.89 \mathrm{~mm}$ & $1.54 \mathrm{~mm}$ & $1.61 \mathrm{~mm}$ \\
\hline Difference $(\delta)$ & $0.46 \mathrm{~mm}$ & $0.33 \mathrm{~mm}$ & $0.49 \mathrm{~mm}$ & $0.96 \mathrm{~mm}$ & $0.76 \mathrm{~mm}$ & $1.04 \mathrm{~mm}$ \\
\hline Rank & 2 & 3 & 1 & 2 & 3 & 1 \\
\hline
\end{tabular}

\subsection{Hole surface roughness}

\subsubsection{Main distribution of the hole $\boldsymbol{R}_{\boldsymbol{a}}$ from the CNC and Kuka systems}

Figure 12 shows the surface roughness for all of the drilled holes. The mean measured surface roughness $R_{a}$ for all the CNC holes is $3.22 \mu \mathrm{m}$. The minimum observed $R_{a}$ is $1.967 \mu \mathrm{m}$ from experiment 4 , and a $\delta$ of $1.17 \mu \mathrm{m}$ can be expected with this setting. The maximum is $4.7 \mu \mathrm{m}$ from experiment 9; this experiment has a large deviation as does experiments 1,5 , and 7 ( $\delta$ of $1.1-1.4 \mu \mathrm{m})$. There is no consistency within the 9 experiments.

The mean measured $R_{a}$ for all the Kuka drilled holes show that the mean roughness is $4.00 \mu \mathrm{m}$. The minimum observed $R_{a}$ is $2.13 \mu \mathrm{m}$ from experiment 3 ; this also has a low distribution $(\delta$ of $0.59 \mu \mathrm{m})$ making it the optimum setting. The maximum is $5.68 \mu \mathrm{m}$ from experiment 9 . Experiments 2 and 4 had wide deviations making them less predictable settings for consistent surface roughness.

There is no consistency in the experiments and both systems have a wide deviation in results, with experiments 2 and
4 for the Kuka system producing the widest deviations making them unsuitable settings for drilling with a consistent surface roughness. An important observation to be made when considering both systems is that although the average $R_{a}$ for the CNC system is better throughout the 9 experiments (>19.5\%), the optimized Kuka system can produce a comparable surface finish (experiment 3 ) to the best observed $R_{a}$ drilled by the CNC system (experiment 4). This result is similar to the exit burr height results and suggests that $R_{a}$ may not be significantly influenced by the overall stiffness of the systems.

\subsubsection{Main effects of the hole $\boldsymbol{R}_{\boldsymbol{a}}$ from the CNC and Kuka systems}

Table 4 and Figs. 13 and 14 show the main effects of the parameters on the hole surface finish for both drill systems. Based on the results for the CNC drilled holes the $S_{s}$ is the most influential factor; as the speed is increased from 2000 to 3000 RPM the roughness of the hole increases and a $\delta$ of $1.17 \mu \mathrm{m}$ can be expected. The $P_{c}$ is the second most important and the results show that the surface roughness improves with 4 passes. For the Kuka system the $P_{c}$ has the largest
Fig. 10 Main effect plot for burr heights for $\mathrm{CNC}$ drilled holes

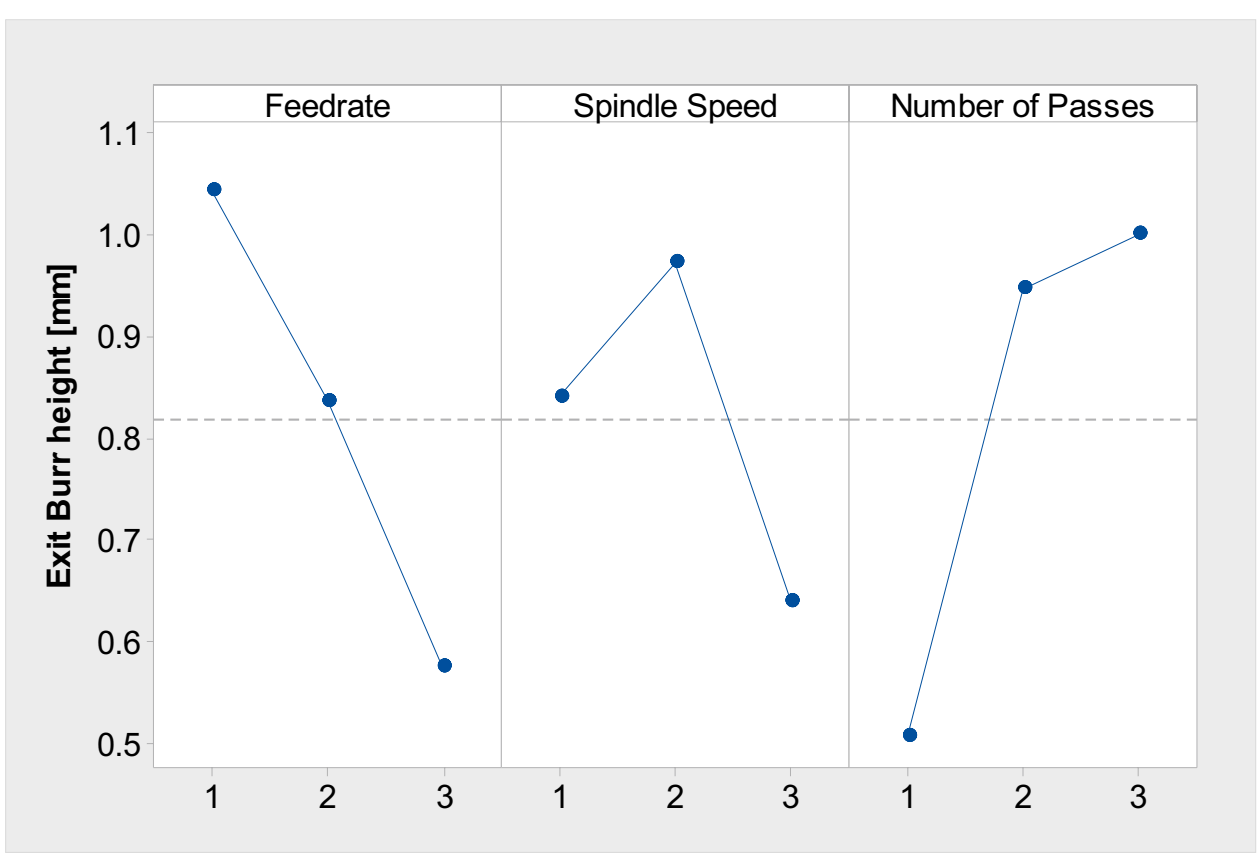


Fig. 11 Main effect plot for burr heights for Kuka drilled holes

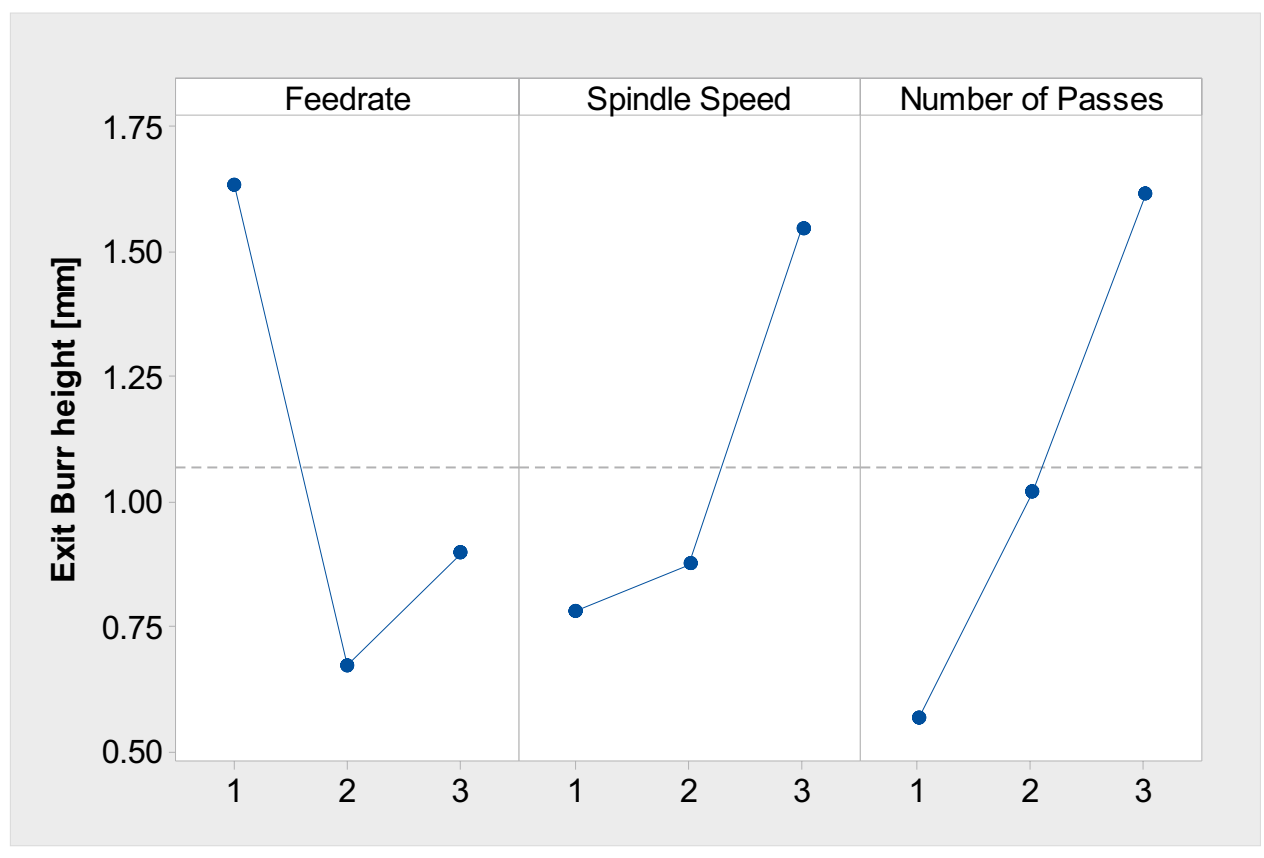

influence with a $\delta$ of $2.1 \mu \mathrm{m}$ observed. However, the result is not straightforward as the surface roughness is at $3.49 \mu \mathrm{m}$ for 2 passes but deteriorates to $5.31 \mu \mathrm{m}$ at three passes and then improves again at 4 passes with a $R_{a}$ of $3.2 \mu \mathrm{m}$. This behavior could be due to interactions with the other factors. The second most influential factor is $S_{s}$. As the $S_{s}$ is increased the surface roughness improves ( $\delta$ of $1.22 \mu \mathrm{m}$ ); this change is the complete opposite to that observed with the CNC and could be due to increased vibrations experienced by the Kuka resulting in a lower surface finish.

\section{Optimized solutions for the Kuka system}

\subsection{Factor interactions of the exit burr heights}

The main effects are instructive when optimizing a process; however, there are often interactions between the factors that can be less easy to predict. Using ANOVA it was possible to plot interactions for the exit burr heights (Fig. 15). In Fig. 15a there is an interaction observed with low $F_{r}$ and $P_{c}$. It can be seen that the optimum setting for

Fig. 12 Surface roughness for CNC and Kuka drilled holes

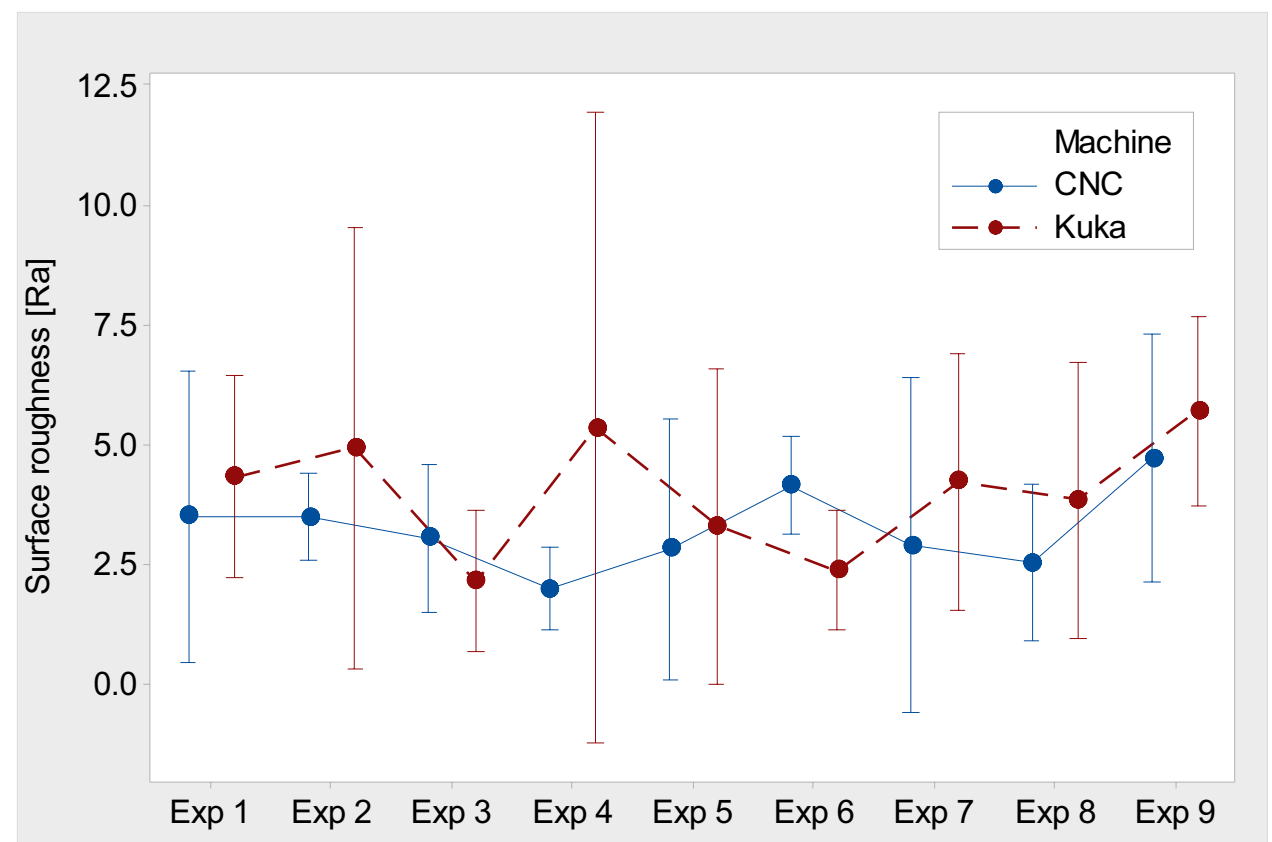


Table 4 Surface roughness main effect results

\begin{tabular}{|c|c|c|c|c|c|c|}
\hline \multirow[t]{2}{*}{ Level } & \multicolumn{3}{|l|}{$\mathrm{CNC}$} & \multicolumn{3}{|l|}{ Kuka } \\
\hline & $F_{r}$ & $S_{s}$ & $P_{c}$ & $F_{r}$ & $S_{s}$ & $P_{c}$ \\
\hline 1 & $3.33 \mu \mathrm{m}$ & $2.77 \mu \mathrm{m}$ & $3.37 \mu \mathrm{m}$ & $3.78 \mu \mathrm{m}$ & $4.61 \mu \mathrm{m}$ & $3.49 \mu \mathrm{m}$ \\
\hline 2 & $2.96 \mu \mathrm{m}$ & $2.93 \mu \mathrm{m}$ & $3.38 \mu \mathrm{m}$ & $3.65 \mu \mathrm{m}$ & $4.01 \mu \mathrm{m}$ & $5.31 \mu \mathrm{m}$ \\
\hline 3 & $3.36 \mu \mathrm{m}$ & $3.95 \mu \mathrm{m}$ & $2.90 \mu \mathrm{m}$ & $4.57 \mu \mathrm{m}$ & $3.39 \mu \mathrm{m}$ & $3.20 \mu \mathrm{m}$ \\
\hline Difference $(\delta)$ & $0.40 \mu \mathrm{m}$ & $1.17 \mu \mathrm{m}$ & $0.47 \mu \mathrm{m}$ & $0.92 \mu \mathrm{m}$ & $1.22 \mu \mathrm{m}$ & $2.10 \mu \mathrm{m}$ \\
\hline Rank & 3 & 1 & 2 & 3 & 2 & 1 \\
\hline
\end{tabular}

low height burrs is a low $F_{r}$ and low $P_{c}$; however, if you increase the passes at the same $F_{r}$ there is a significant increase in the burr height. There is no significant interaction for medium and high $F_{r}$.

For the relationship between the $S_{s}$ and $P_{c}$ (Fig. 15b) there is no obvious interaction except for the large increase in burr height observed at the highest $S_{s}$ when moving for 3 passes to 4 . An optimum setting would be 2 passes and a low $S_{s}$. When considering the relationship between the $F_{r}$ and $S_{s}$ (Fig. 15c) the medium and high $F_{r}$ have no significant relationships with $S_{s}$. However, there is a significant interaction with the low $F_{r}$ and $S_{s}$, where the exit burr height increased with the change from a 2000 to 3000 RPM. The setting of a low $F_{r}$ and $S_{s}$ appear optimum for producing low height burrs.

There are three factor interactions that result in height burr heights, a $F_{r}$ of 200 and 4 passes, 3000 RPM and 4 passes, and 3000 RPM and a $F_{r}$ of 200. There is evidence for increased energy at the hole exit and a change in exit velocity. To reduce the drill exit force, there is the option of a final micropeck cycle. This optimized solution will have very little influence on the overall cycle time and the Kuka robot has a high capability to use this function.

\subsection{Factor interactions for surface roughness}

In Fig. 12 the main effect of $P_{c}$ shows that three passes are detrimental to the surface finish. The same result is shown in the interaction plot for $S_{s}$ and $P_{c}$ (Fig. 16a). The best surface finish can be achieved with a low $F_{r}$ and 4 passes followed by a medium federate and 2 passes. It is difficult to ascertain the reason for the influence of the $P_{c}$, and a possible cause could be that three passes produce step effect in the hole. For optimized machine setting it is clear that additional passes should be avoided for particularly with a high $F_{r}$ setting.

Figure 14b shows the relationship between the $S_{s}$ and $P_{c}$. This also shows that 3 passes are detrimental to surface finish particularly with a high spindle speed. However, the high $S_{s}$ setting does produce the better surface quality with 2 passes and 4 passes.

Figure 16c shows that the best surface finish can be achieved with a high $S_{s}$ and low-medium $F_{r}$. There is an
Fig. 13 Main effect plot for surface roughness for $\mathrm{CNC}$ drilled holes

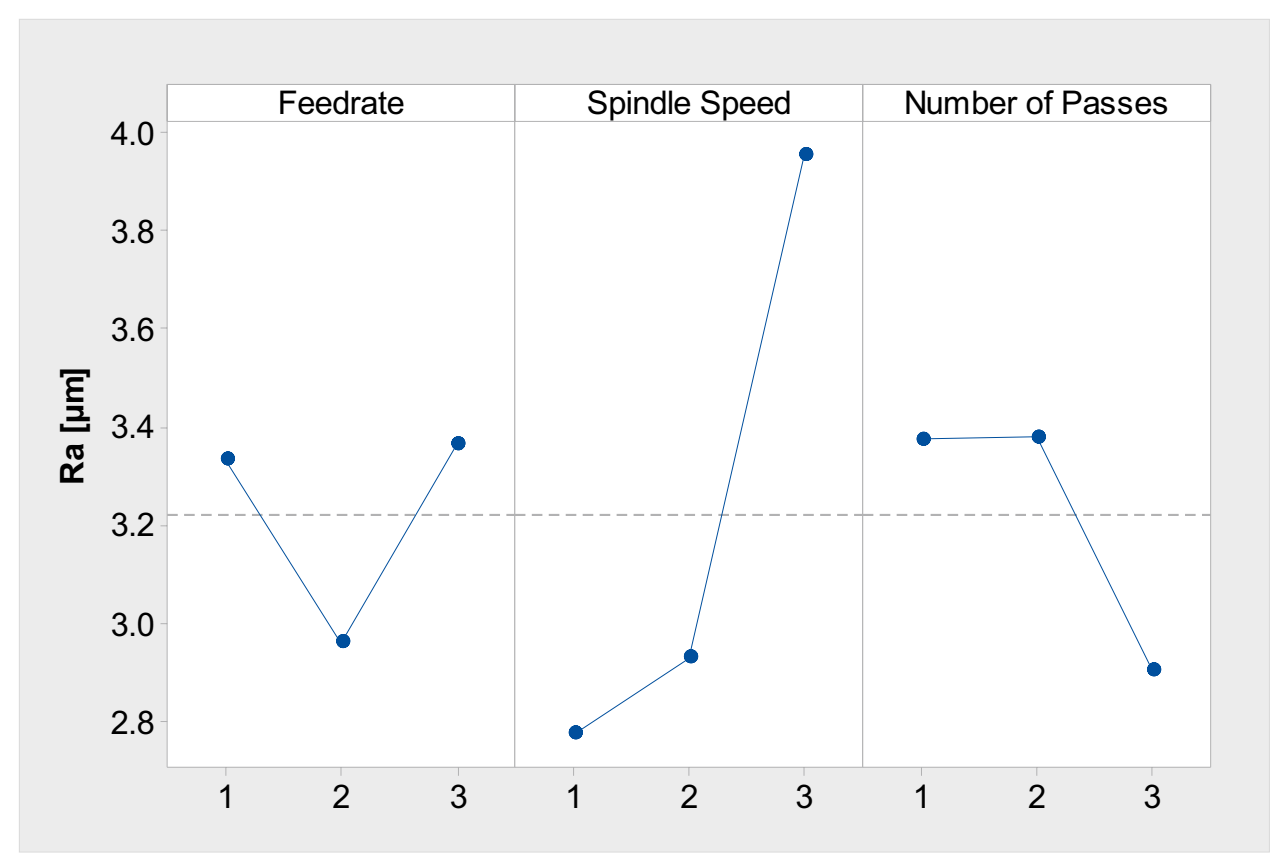


Fig. 14 Main effect plot for surface roughness for Kuka drilled holes

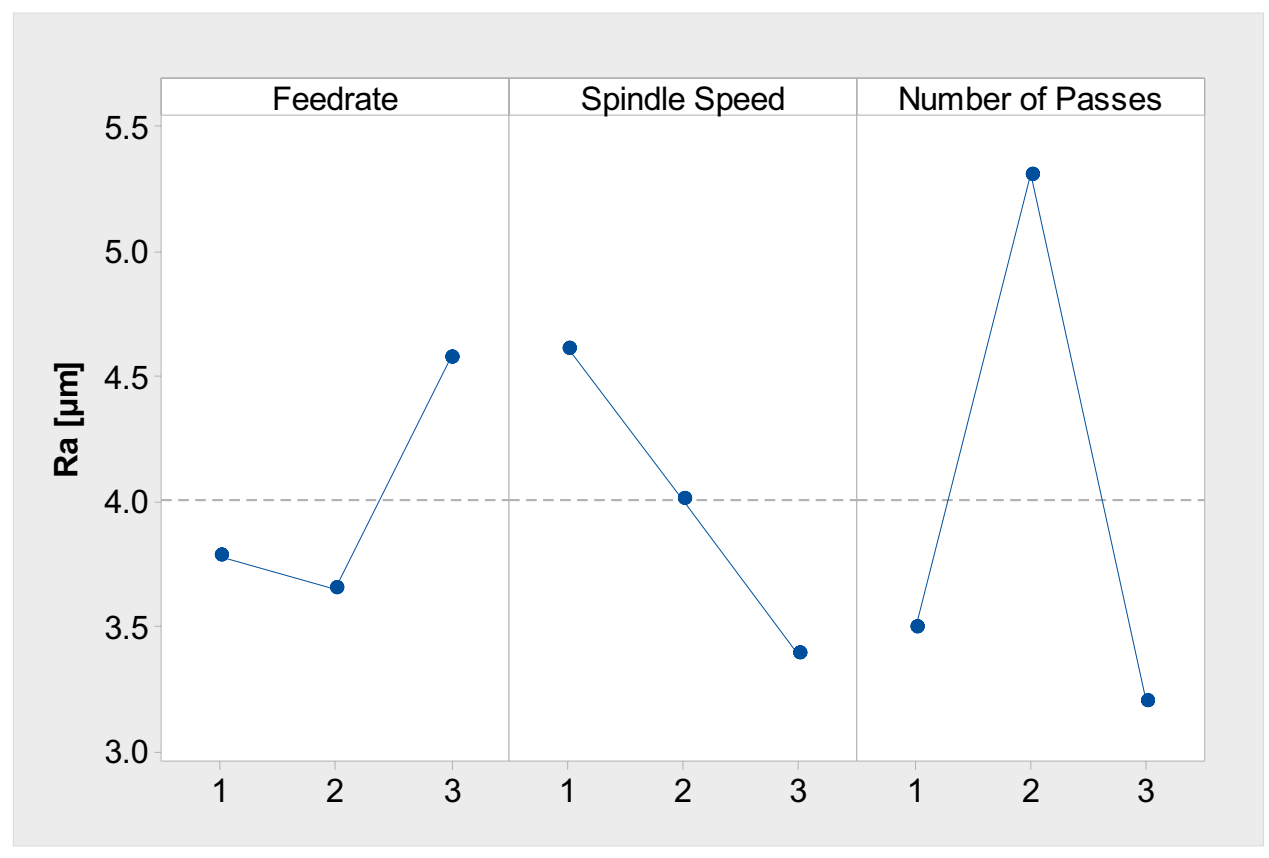

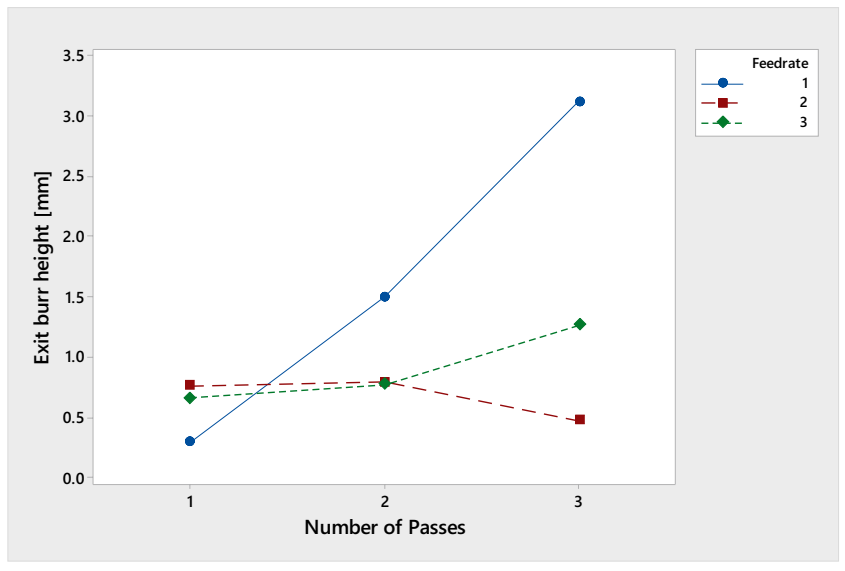

(a)

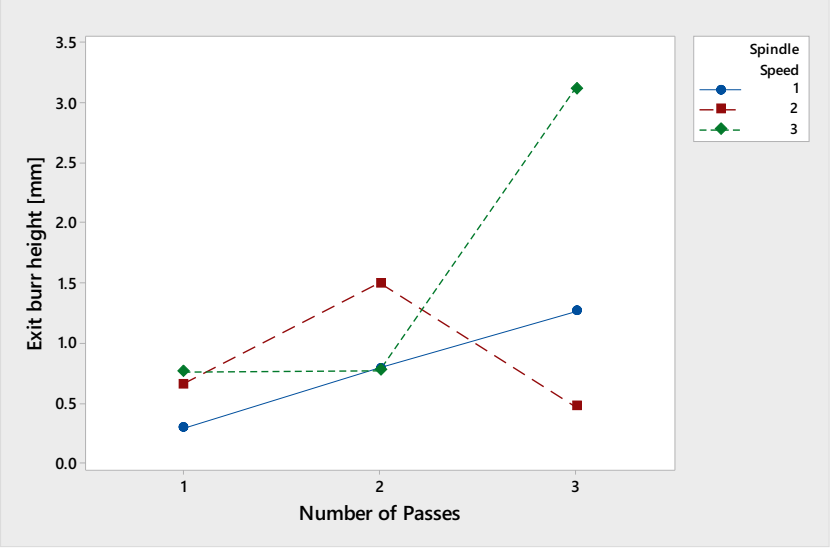

(b)

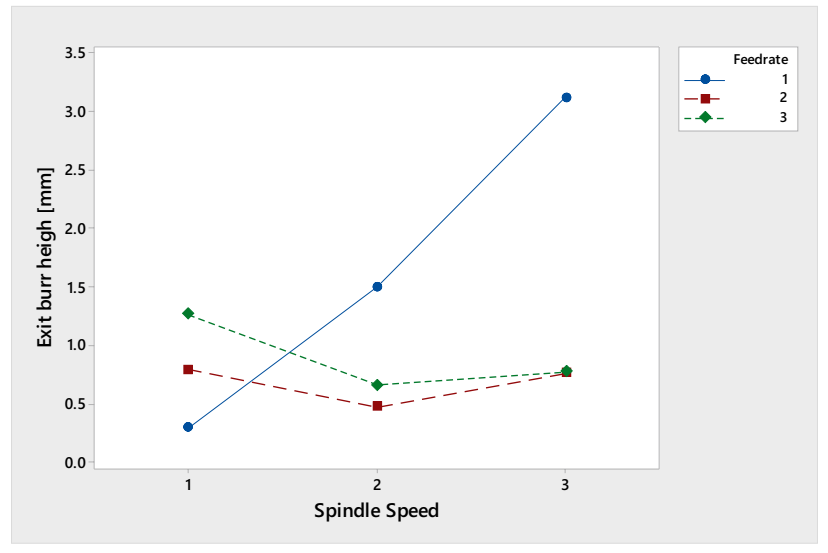

(c)

Fig. 15 Interaction plots for burr heights (a) $P_{c}$ and $F_{r}$, (b) $P_{c}$ and $S_{s}$, and (c) $S_{s}$ and $F_{r}$ 


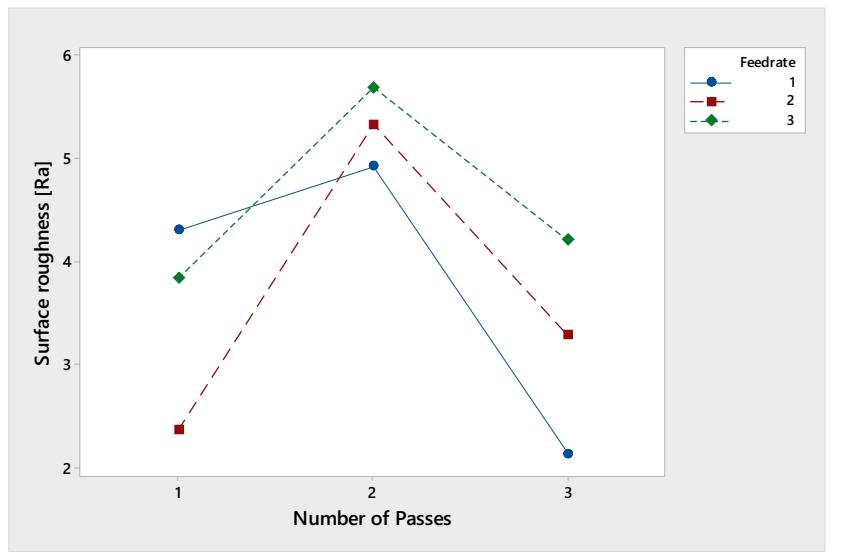

(a)

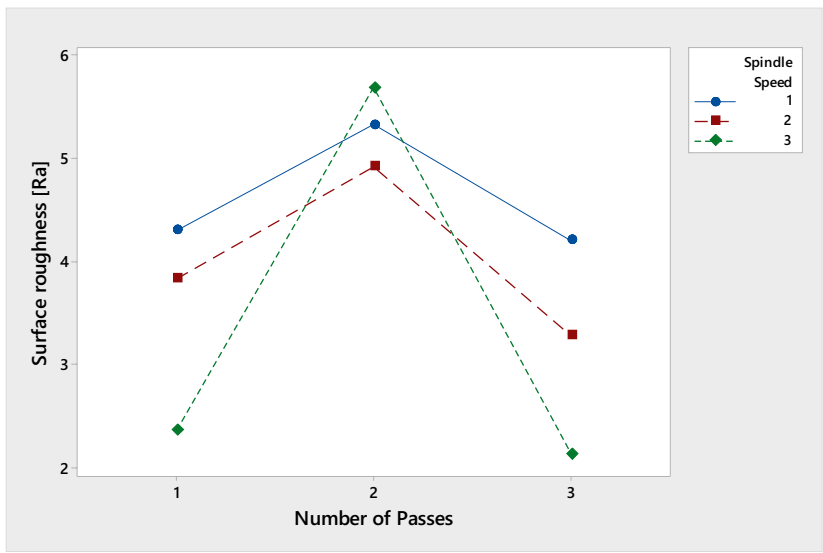

(b)

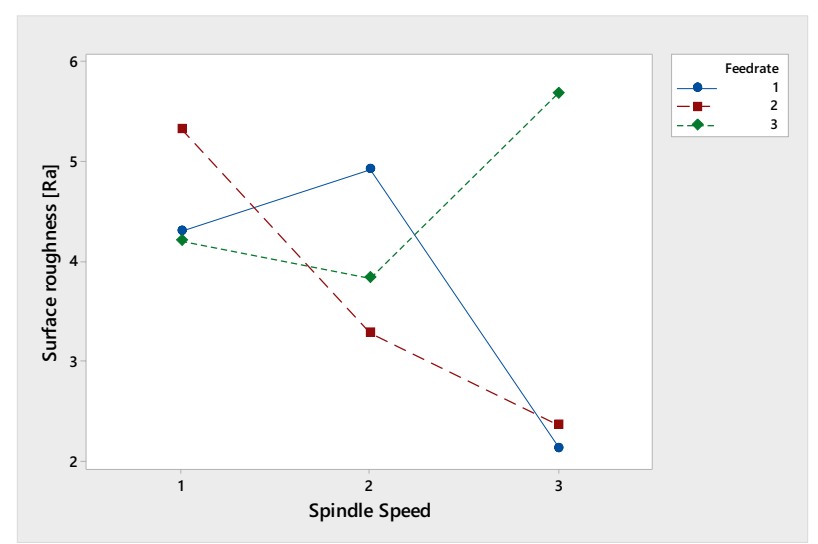

(c)

Fig. 16 Interaction plots for surface roughness (a) $P_{c}$ and $F_{r}$, (b) $P_{c}$ and $S_{s}$, and (c) $S_{s}$ and $F_{r}$

Table 5 Optimized settings

\begin{tabular}{lllll}
\hline Optimum settings & CNC burr height & Kuka burr height & $\begin{array}{l}\text { CNC surface } \\
\text { roughness }\end{array}$ & $\begin{array}{l}\text { Kuka } \\
\text { surface } \\
\text { roughness }\end{array}$ \\
\hline$F_{r}(\mathrm{~mm} / \mathrm{min})$ & 400 & 300 & 300 & 300 \\
$S_{s}(\mathrm{RPM})$ & 3000 & 2000 & 2000 & 3000 \\
$P_{c}$ & 1 & 2 & 4 & 4 \\
Predicted result & $0.86 \mathrm{~mm}$ & $0.114 \mathrm{~mm}$ & $2.20 \mu \mathrm{m}$ & $2.24 \mu \mathrm{m}$ \\
\hline
\end{tabular}

interaction with the high $S_{s}$ and $F_{r}$; in that, it does not follow this trend and actually results in the producing the worst surface finish. In addition, a low $S_{s}$ and medium $F_{r}$ provide a poor surface finish. The low $S_{s}$ is shown to produce poor surface quality for all $F_{r}$ settings, and the high $S_{s}$ can result in the highest quality for low to medium $F_{r}$ setting. The anomaly of a high $S_{s}$ and $F_{r}$ producing the worst surface finish could be due to the $F_{r}$ exceeding the cutting capability and providing an increase of force at the cutting edges of the drill.

\subsection{Predicted results}

The best quality hole for the purpose of this research is defined as lowest surface roughness and lowest exit burr height. An analysis was conducted on the Taguchi L9 array 
which included all responses from the experimental data. The focus of the research was also to observe if the Kuka drilling system could be optimized in a way that the hole quality could be comparable with that of a CNC machine.

The results for both systems show that there is no single experiment that provides a low surface roughness and low exit burr height and that for both systems postprocessing will be required for the holes to meet aerospace drilling specifications. The Kuka experimental data shows that the optimum hole providing the lowest $R_{a}$ value is experiment $3(2.13 \mu \mathrm{m})$ and this was with a $F_{r}$ of $200 \mathrm{~mm} / \mathrm{min}, S_{s}$ of $3000 \mathrm{RPM}$, and a $P_{c}$ with 4 passes through the workpiece. Experiment 1 produced the lowest exit burr height value $(0.29 \mathrm{~mm})$ and this was with a $F_{r}$ of $200 \mathrm{~mm} / \mathrm{min}, S_{s}$ of $2000 \mathrm{RPM}$, and 2 passes through the workpiece. A compromise for the exit burr height and surface roughness would be experiment number 6 where the measured heights and $R_{a}$ are $0.77 \mathrm{~mm}$ and $2.35 \mu \mathrm{m}$, respectively.

Using the main effect plots (Figs. 11 and 14), it is possible to take the factor settings that provide an optimum result and predict the average response for this combination of control factor levels. The objective is to determine the settings that minimize the means of the burr height and $R_{a}$. The results in Table 5 show that the burr height can see an improvement of $60.68 \%$ when compared to the measured response from experiment 1 . For the surface roughness no improvement is predicted and experiment 3 remains the preferred setting.

\section{Conclusion}

This paper investigated the capability of a highly flexible industrial robot modified with a high-speed machine spindle for drilling. Experiments were conducted using the Taguchi design methodology to determine the optimum $8 \mathrm{~mm}$ through drilling parameters while machining aluminum 6061-T6. Drilling was conducted using CNC and Kuka systems and the hole surface roughness and exit burr heights were the quality characteristics of importance. For both systems the main control factors were $F_{r}, S_{s}$, and the $P_{c}$. Prior to the experiments vibration tests were undertaken within the Kuka KR16 robot workspace to identify the spindle speeds and workpiece locations with the minimal vibrations. The key findings from the experiments undertaken were the following:

- Identifying the correct robot pose for drilling is important for machine optimization; in order to do this, a state-ofthe-art condition monitoring system was set up on the end effector spindle of the Kuka system to establish the vibrations experienced during operation. Within the drilling workspace 99 different pose positions were measured. The initial tests were conducted without the spindle on in order to establish which robot pose had increased stiffness; this was then followed by the same positions but at different $S_{s}$. The results show that due to the kinematic chain of 6-axis articulated robots certain poses within the workspace are stiffer and that the spindle must be active to establish this. By condition monitoring $S_{s}$ and workspaces, robot engineers can improve the capability of their automated drilling systems.

- To establish the capability of the Kuka system a design of experiment approach was used identify which of the selected parameters influenced burr height. The system was also benchmarked against a CNC drilling setup. As expected the $\mathrm{CNC}$ was capable of producing the lowest burr heights. However, the Kuka matched and outperformed the $\mathrm{CNC}$ in 6 of the experiments and the distribution was better overall. For both systems postprocess deburring would be required to meet aerospace manufacture standards.

- For the Kuka system, the number of passes of the $P_{c}$ was established as the most influential process factor on reducing the burr height. Using ANOVA it was possible to identify interactions, and the setting of a low $F_{r}$ and $S_{s}$ can be used for producing low height burrs. Overall the results show that there is much scope for optimizing the process to control exit burr height and further work should be conducted to control the drill exit forces on the final pecking cycle.

- The selected control factors had a large effect on both the Kuka and the CNC systems. An important observation is that the CNC system produces better surface quality, but there are two settings of the Kuka that outperform the much stiffer CNC machine. For the Kuka system $P_{c}$ has the largest influence followed by $S_{s}$. The main effect of $P_{c}$ shows that additional passes are detrimental to the surface finish and the ANOVA interaction shows that this should be avoided particularly with a high $F_{r}$ setting.

By using a condition monitoring and a design of experiment approach it has been possible to identify a range of quality standards that can be achieved using a state-of-theart robotic machining system. The system is far more flexible than a CNC milling machine, and when benchmarked against a CNC, the Kuka performs well when drilling aerospace aluminum. Optimization of selected parameter has been shown to improve the hole surface roughness and reduce exit burrs. With further research there is also potential for further improvements to be made, particularly with control of the drill exit force and control of the pecking cycles while drilling.

Acknowledgements The authors would like to acknowledge the support of the Future Manufacturing Research Institute, College of Engineering, Swansea University and Advanced Sustainable Manufacturing 
Technologies (ASTUTE 2022) project, which is partly funded from the EU's European Regional Development Fund through the Welsh European Funding Office, in enabling the research upon which this paper is based. Further information on ASTUTE can be found at www. astutewales.com.

Author contribution B. Pereira - principal researcher, conducted $60 \%$ of the tests; C. Griffiths - initial proposal and conducted Minitab analysis of test results; B. Birch — conducted experiments and drilling setup support; and A. Rees - verification of test results.

Availability of data and material Data is held and available.

Code Availability Not applicable.

\section{Declarations}

Ethics approval Not applicable.

Consent to participate All authors consent to participate.

Consent for publication All authors consent to publish.

Conflict of interest The authors declare no competing interests.

Open Access This article is licensed under a Creative Commons Attribution 4.0 International License, which permits use, sharing, adaptation, distribution and reproduction in any medium or format, as long as you give appropriate credit to the original author(s) and the source, provide a link to the Creative Commons licence, and indicate if changes were made. The images or other third party material in this article are included in the article's Creative Commons licence, unless indicated otherwise in a credit line to the material. If material is not included in the article's Creative Commons licence and your intended use is not permitted by statutory regulation or exceeds the permitted use, you will need to obtain permission directly from the copyright holder. To view a copy of this licence, visit http://creativecommons.org/licenses/by/4.0/.

\section{References}

1. Wang YQ, Bao YJ, Gao H (2007) Studies on disfigurementfee technology of drilling carbon fiber reinforced plastics. In: Advanced Materials Research. vol. 24. Trans Tech Publ 125-132

2. Boeing Frontiers Online. 109380-Leslie Nichols;. Available from: https://www.boeing.com/news/frontiers/archive/2004/September

3. Bringmann E, Kramer A (2008) Model-based testing of automotive systems. In, (2008) 1st international conference on software testing, verification, and validation. IEEE 2008:485-493

4. Phillips M (1999) Agile manufacturing in the aerospace industry: an industrial viewpoint. Int J Agil Manag Syst 1(1):17-22

5. Boeing. 109380-Leslie Nichols;. Available from: https://www. boeing.com/commercial/market/commercial-market-outlook/

6. Airbus Global Market Forecast 2018-2037; . Available from: https://www.airbus.com/aircraft/market/global-market-forecast. html

7. Jaacks K (2016) Automated circumferential joint assembly in aircraft productions

8. Frommknecht A, Kuehnle J, Effenberger I, Pidan S (2017) Multisensor measurement system for robotic drilling. Robot Comput Integr Manuf 47:4-10
9. Campbell Jr FC (2011) Manufacturing technology for aerospace structural materials. Elsevier

10. Duquette J, Lortie M, Rossignol M (1997) Perception of difficulties for the back related to assembly work: general findings and impact of back health. Appl Ergon 28(5-6):389-396

11. Menegon F, Fischer F (2012) Musculoskeletal reported symptoms among aircraft assembly workers: a multifactorial approach. Work 41:3738-3745

12. Tian W, Zhou W, Zhou W, Liao W, Zeng Y (2013) Auto-normalization algorithm for robotic precision drilling system in aircraft component assembly. Chin J Aeronaut 26(2):495-500

13. Shi Z, Yuan P, Wang Q, Chen D, Wang T (2016) New design of a compact aero-robotic drilling end effector: an experimental analysis. Chin J Aeronaut 29(4):1132-1141

14. Liu H, Zhu W, Dong H, Ke Y (2017) A helical milling and oval countersinking end-effector for aircraft assembly. Mechatronics 46:101-114

15. Ralph W, Johnson W, Makeev A, Newman J (2007) Fatigue performance of production-quality aircraft fastener holes. Int $\mathbf{J}$ Fatigue 29(7):1319-1327

16. Coleman R (1991) The effects of design, manufacturing processes and operations management on the assembly of aircraft composite structure

17. Zhang H, Wang J, Zhang G, Gan Z, Pan Z, Cui H et al (2005) Machining with flexible manipulator: toward improving robotic machining performance. In: Advanced Intelligent Mechatronics. Proceedings, 2005 IEEE/ASME International Conference on. IEEE 1127-1132

18. Tao J, Qin C, Liu C (2019) A synchro extracting-based method for early chatter identification of robotic drilling process. Int $\mathbf{J} \mathrm{Adv}$ Manuf Technol 100(1-4):273-285

19. Munoa J, Beudaert X, Dombovari Z, Altintas Y, Budak E, Brecher $\mathrm{C}$ et al (2016) Chatter suppression techniques in metal cutting. CIRP Ann 65(2):785-808

20. Quintana G, Ciurana $\mathbf{J}$ (2011) Chatter in machining processes: a review. Int J Mach Tools Manuf 51(5):363-376

21. Shao Y, Deng X, Yuan Y, Mechefske CK, Chen Z (2014) Characteristic recognition of chatter mark vibration in a rolling mill based on the non-dimensional parameters of the vibration signal. J Mech Sci Technol 28(6):2075-2080

22. Balaji M, Venkata Rao K, Mohan Rao N, Murthy B (2018) Optimization of drilling parameters for drilling of TI-6Al-4V based on surface roughness, flank wear and drill vibration. Measurement 114:332-339

23. Zhang JZ, Chen JC (2009) Surface roughness optimization in a drilling operation using the Taguchi design method. Mater Manuf Processes 24(4):459-467

24. Chen Y, Dong F (2013) Robot machining: recent development and future research issues. Int J Adv Manuf 66(9-12):1489-1497

25. Huang MF, Lin TR (2004) Application of grey-Taguchi method to optimise drilling of aluminium alloy 6061 with multiple performance characteristics. Mater Sci Technol 20(4):528-532

26. Bahçe E, Özdemir B (2019) Investigation of the burr formation during the drilling of free-form surfaces in al 7075 alloy. J Mater Res Technol 8(5):4198-4208

27. Chern GL (1993) Analysis of burr formation and breakout in metal cutting (Doctoral dissertation, University of California, Berkeley)

28. Kishawy H, Becze C, McIntosh D (2004) Tool performance and attainable surface quality during the machining of aerospace alloys using self-propelled rotary tools. J Mater Process Technol 152(3):266-271

29. Tian W, Hu J, Liao W, Bu Y, Zhang L (2016) Formation of interlayer gap and control of interlayer burr in dry drilling of stacked aluminum alloy plates. Chinese J Aeronaut 29(1):283-291 
30. Costa ES, Da Silva MB, Alisson Machado AR (2009) Burr produced on the drilling process as a function of tool wear and lubricant-coolant conditions, FEMEC, Uberlandia, Brazil. J Braz Soc Mech Sci Eng vol.31 no.1 Rio de Janeiro Jan./Mar. 2009

31. Pilný L, De Chiffre L, Posca M, Villumsen M (2012) Hole quality and burr reduction in drilling aluminium sheets. CIRP J Manuf Sci Technol 5(2):102-107

32. Jie L (2013) The formation and effect of interlayer gap in dry drilling of stacked metal materials. Int J Adv Manuf 69(5-8):1263-1272.

33. Yoomin Ahn \& Seoung Hwan Lee (2017) Classification and prediction of burr formation in micro drilling of ductile metals. Int $\mathbf{J}$ Prod Res 55(17):4833-4846. https://doi.org/10.1080/00207543. 2016.1254355

34. Durham BJ (2014) Determining appropriate levels of robotic automation in commercial aircraft nacelle assembly. Massachusetts Institute of Technology

35. Bi S, Liang J (2010) Robotic drilling system for titanium structures. Int J Adv Manuf Technol 54(5-8):767-774

36. Ljasenko S, Lohse N, Justham L (2016) A comparison of the manufacturing resilience between fixed automation systems and mobile robots in large structure assembly. Procedia CIRP 57:235-240

37. Gates D. Boeing Abandons Its Failed Fuselage Robots on the $777 \mathrm{X}$, Handing the Job Back to Machinists. Seatle Times.
Available from: https://www.seattletimes.com/business/boeingaerospace/boeing-abandonsits-failed-fuselage-robots-on-the777x-handing-the-job-back-tomachinists/. [Accessed 27th April 2020]

38. Bu Y, Liao W, Tian W, Zhang J, Zhang L (2017) Stiffness analysis and optimization in robotic drilling application. Precis Eng 49:388-400

39. de Aguiar M, Diniz A, Pederiva R (2013) Correlating surface roughness, tool wear and tool vibration in the milling process of hardened steel using long slender tools. Int J Mach Tools Manuf 68:1-10

40. Kataoka R, Shamoto E (2019) Influence of vibration in cutting on tool flank wear: fundamental study by conducting a cutting experiment with forced vibration in the depth-of-cut direction. Precis Eng 55:322-329

41. Diaz Posada JR, Schneider U, Pidan S, Gersvand M, Stelzer P, Verl A (2016) High accurate robotic drilling with external sensor and compliance model-based compensation. IEEE

Publisher's Note Springer Nature remains neutral with regard to jurisdictional claims in published maps and institutional affiliations. 